\title{
COMMON FIXED POINT THEOREMS FOR CONTRACTION TYPE MAPPINGS IN PARTIALLY ORDERED METRIC SPACES
}

\author{
CHI-MING CHEN AND CHING-JU LIN
}

\begin{abstract}
The purpose of this paper is to present common fixed point theorems for a stronger Meir-Keeler type mapping in partially ordered metric space. Our results generalize some recent results.
\end{abstract}

\section{Introduction and Preliminaries}

Throughout this paper, by $\Re^{+}$we denote the set of all nonnegative numbers, while $\mathscr{N}$ is the set of all natural numbers. Existence of fixed point for contraction type maps in partially ordered sets has been considered recently in $[1,2,3,4,5,6,7,10]$.

In this paper, we define a stronger Meir-Keeler type mapping $\psi: \Re^{+} \rightarrow[0,1)$ and we present a common fixed point theorem for this generalized nonlinear contraction in partially ordered complete metric spaces.

In 1973, Geraghty [8] introduced the following generalization of Banach's contraction principle.

Let $S$ denote the class of the functions $\beta: \Re^{+} \rightarrow[0,1)$ which satisfy the condition

$$
\beta\left(t_{n}\right) \rightarrow 1 \Rightarrow t_{n} \rightarrow 0
$$

Theorem 1 ([8]). Let $(X, d)$ be a complete metric space, and let $f: X \rightarrow X$ be a mapping satisfying

$$
d(f x, f y) \leq \beta(d(x, y)) \cdot d(x, y), \text { for } x, y \in X,
$$

where $\beta \in S$. Then $f$ has a unique fixed point $z \in X$ and $\left\{f^{n}(x)\right\}$ converges to $z$ for each $x \in X$.

Recently, A. Amini-Harandi and H. Emami[3] proved a version of Theorem 1 in the context of complete partially ordered metric spaces, as follows:

Corresponding author: Chi-Ming Chen.

2010 Mathematics Subject Classification. 47H10, 54C60, 54H25, 55M20.

Key words and phrases. Complete partially ordered space, common fixed point theorem, stronger Meir-

Keeler type mapping.

*Research supported by the NSC. 
Theorem 2 ([3]). Let $(X, \preceq)$ be a partially ordered set and suppose that there exists a metric d in $X$ such that $(X, d)$ is a complete metric space. Let $f: X \rightarrow X$ be an increasing mapping such that there exists an element $x_{0} \in X$ with $x_{0} \leq f x_{0}$. Suppose that there exists $\beta \in S$ such that

$$
d(f x, f y) \leq \beta(d(x, y)) \cdot d(x, y), \text { for } x, y \in X \text { with } x \geq y \text {. }
$$

Assume that either $f$ is continuous or $X$ is such that

$$
\text { if an increasing sequence }\left\{x_{n}\right\} \rightarrow x \text { in } X \text {, then } x_{n} \leq x, \forall n \text {. }
$$

Besides, if

$$
\text { for each } x, y \in X \text { there exists } z \in X \text { which is comparable to } x \text { and } y \text {. }
$$

Then $f$ has a unique fixed point.

We recall the notion of the Meir-Keeler type mapping. A function $\psi: \Re^{+} \rightarrow \Re^{+}$is said to be a Meir-Keeler type mapping(see [9]), if for each $\eta \in \Re^{+}$, there exists $\delta>0$ such that for $t \in \Re^{+}$with $\eta \leq t<\eta+\delta$, we have $\psi(t)<\eta$. We now define a new stronger Meir-Keeler conetype mapping, as follows:

Definition 1. A mapping $\psi: \Re^{+} \rightarrow[0,1)$ is called a stronger Meir-Keeler type mapping, if for each $\eta>0$, there exists $\delta>0$ such that for $t \in \Re^{+}$with $\eta \leq t<\delta+\eta$, there exists $\gamma_{\eta} \in[0,1)$ such that $\psi(t)<\gamma_{\eta}$.

Example 1. Le $\psi: \Re^{+} \rightarrow[0,1)$ be defined as $\psi(t)=\frac{t}{t+1}$, then $\psi$ is a stronger Meir-Keeler type mapping.

\section{Main results}

Definition 2 ([4]). Let $(X, \preceq)$ be a partially ordered set and $f, g: X \rightarrow X$. Then $f$ is said to be $g$-nondecreasing if for $x, y \in X$,

$$
g x \leq g y \Longrightarrow f x \leq f y .
$$

In the sequel, we prove the main result of this paper.

Theorem 3. Let $(X, \preceq)$ be a partially ordered set and suppose that there exists a metric $d$ in $X$ such that $(X, d)$ is a complete metric space. Let $f, g: X \rightarrow X$ be such that $f X \subset g X$, and $f$ be a g-nondecreasing mapping. Suppose that there exists a stronger Meir-Keeler type mapping $\psi: \Re^{+} \rightarrow[0,1)$ such that

$$
d(f x, f y) \leq \psi(d(g x, g y)) \cdot d(g x, g y), \text { for } x, y \in X \text { with } g x \geq g y .
$$


Also suppose,

$$
\begin{aligned}
& \text { if }\left\{g x_{n}\right\} \subset X \text { is a nondecreasing sequence with } g x_{n} \rightarrow g z \in g X, \\
& \text { then } g x_{n} \leq g z \text { and } g z \leq g^{2} z \text { for all } n \in \mathscr{N} .
\end{aligned}
$$

Suppose also that $g X$ is closed. If there exists an $x_{0} \in X$ with $g x_{0} \leq f x_{0}$, then $f$ and $g$ have a coincidence point.

Further, if $f, g$ commute at their coincidence points, then $f$ and $g$ have a common fixed point in $X$.

Proof. Given $x_{0} \in X$ such that $g x_{0} \leq f x_{0}$. Since $f X \subset g X$, we can choose $x_{1} \in X$ such that $g x_{1}=f x_{0}$. Continuing this process, we define the sequence $\left\{x_{n}\right\}$ in $X$ recursively as follows:

$$
f x_{n}=g x_{n+1} \text {, for all } n \in \mathscr{N} \text {. }
$$

Since $g x_{0} \leq f x_{0}$ and $f$ is a $g$-nondecreasing mapping, we have

$$
f x_{0} \leq f x_{1} \leq f x_{2} \cdots \leq f x_{n} \leq f x_{n+1} \leq \cdots
$$

In what follows we will suppose that $d\left(f x_{n}, f x_{n+1}\right)>0$ for all $n \in \mathscr{N}$, since if $f x_{n+1}=f x_{n}$ for some $n$, then $f x_{n+1}=g x_{n+1}$, that is, $f$ and $g$ have a coincidence point $x_{n+1}$, and so we complete the proof.

By (1), we have

$$
\begin{aligned}
d\left(f x_{n}, f x_{n+1}\right) & \leq \psi\left(d\left(g x_{n}, g x_{n+1}\right)\right) \cdot d\left(g x_{n}, g x_{n+1}\right) \\
& \leq d\left(g x_{n}, g x_{n+1}\right) \\
& \leq d\left(f x_{n-1}, f x_{n}\right) .
\end{aligned}
$$

Then the sequence $\left\{d\left(f x_{n+1}, f x_{n}\right)\right\}$ is descreasing and bounded below. Let $\lim _{n \rightarrow \infty}$ $d\left(f x_{n+1}, f x_{n}\right)=\eta \geq 0$. Then there exists $\kappa_{0} \in \mathscr{N}$ and $\delta>0$ such that for all $n>\kappa_{0}$

$$
\eta \leq d\left(f x_{n+1}, f x_{n}\right)<\eta+\delta
$$

For each $n \in \mathscr{N}$, since $\psi: \Re^{+} \rightarrow[0,1)$ is a stronger Meir-Keeler type mapping, for these $\eta$ and $\delta>0$ we have that for $d\left(f x_{\kappa_{0}+n+1}, f x_{\kappa_{0}+n}\right) \in \Re^{+}$with $\eta \leq d\left(f x_{\kappa_{0}+n+1}, f x_{\kappa_{0}+n}\right)<\delta+\eta$, there exists $\gamma_{\eta} \in[0,1)$ such that $\psi\left(d\left(f x_{\kappa_{0}+n+1}, f x_{\kappa_{0}+n}\right)\right)<\gamma_{\eta}$. Thus, by $(*)$, we can deduce

$$
\begin{aligned}
d\left(f x_{\kappa_{0}+n+1}, f x_{\kappa_{0}+n}\right) & =\psi\left(d\left(f x_{\kappa_{0}+n}, f x_{\kappa_{0}+n-1}\right)\right) \cdot d\left(f x_{\kappa_{0}+n}, f x_{\kappa_{0}+n-1}\right) \\
& <\gamma_{\eta} d\left(f x_{\kappa_{0}+n}, f x_{\kappa_{0}+n-1}\right),
\end{aligned}
$$


and it follows that for each $n \in \mathscr{N}$

$$
\begin{aligned}
d\left(f x_{\kappa_{0}+n+1}, f x_{\kappa_{0}+n}\right) & <\gamma_{\eta} d\left(f x_{\kappa_{0}+n}, f x_{\kappa_{0}+n-1}\right) \\
& <\cdots \\
& <\gamma_{\eta}^{n} d\left(f x_{\kappa_{0}+1}, f x_{\kappa_{0}}\right) .
\end{aligned}
$$

So

$$
\lim _{n \rightarrow \infty} d\left(f x_{\kappa_{0}+n+1}, f x_{\kappa_{0}+n}\right)=0 \text {, since } \gamma_{\eta}<1 \text {. }
$$

We now claim that $\lim _{n \rightarrow \infty} d\left(f x_{\kappa_{0}+m}, f x_{\kappa_{0}+n}\right)=0$ for $m>n$. For $m, n \in \mathscr{N}$ with $m>n$, we have

$$
d\left(f x_{\kappa_{0}+m}, f x_{\kappa_{0}+n}\right) \leq \sum_{i=n}^{m-1} d\left(f x_{\kappa_{0}+i+1}, f x_{\kappa_{0}+i}\right)<\frac{\gamma_{\eta}^{m-1}}{1-\gamma_{\eta}} d\left(f x_{\kappa_{0}+1}, f x_{\kappa_{0}}\right),
$$

and hence $d\left(f x_{m}, f x_{n}\right) \rightarrow 0$, since $0<\gamma_{\eta}<1$. So $\left\{f x_{n}\right\}$ is a Cauchy sequence.

Since $g X$ is closed and $\left\{f x_{n}\right\}=\left\{g x_{n+1}\right\} \subset g X$, there exists $\mu \in X$ such that

$$
\lim _{n \rightarrow \infty} g x_{n}=g \mu=\lim _{n \rightarrow \infty} f x_{n} .
$$

Now we claim that $\mu$ is a coincidence point of $f$ and $g$. Using (1), we have

$$
\begin{aligned}
d(g \mu, f \mu) & \leq d\left(g \mu, f x_{n}\right)+d\left(f x_{n}, f \mu\right) \\
& \leq d\left(g \mu, f x_{n}\right)+\psi\left(d\left(g x_{n}, g \mu\right)\right) \cdot d\left(g x_{n}, g \mu\right) \\
& <d\left(g \mu, f x_{n}\right)+d\left(g x_{n}, g \mu\right) .
\end{aligned}
$$

Letting $n \rightarrow \infty$, we get $g \mu=f \mu$.

Suppose that $f$ and $g$ commute at $\mu$. Let $v=g \mu=f \mu$. Then

$$
f v=f g \mu=g f \mu=g v .
$$

By (2), since $g \mu \preceq g^{2} \mu=g v, g \mu=f \mu$ and $g v=f v$, we deduce

$$
\begin{aligned}
d(f \mu, f v) & \leq \psi(d(g \mu, g v)) \cdot d(g \mu, g v) \\
& <d(f \mu, f v) .
\end{aligned}
$$

Hence $d(f \mu, f v)=0$, that is $d(v, f v)=0$. Therefore,

$$
f v=g v=v .
$$

So we proved that $f$ and $g$ have a common fixed point in $X$.

By Theorem 3, if $g=I=$ the identity mapping, then we immediate obtain the following corollaries. 
Corollary 1. Let $(X, \preceq)$ be a partially ordered set and suppose that there exists a metric $d$ in $X$ such that $(X, d)$ is a complete metric space. Let $f: X \rightarrow X$ be a nondecreasing mapping. Suppose that there exists a stronger Meir-Keeler type mapping $\psi: \Re^{+} \rightarrow[0,1)$ such that

$$
d(f x, f y) \leq \psi(d(x, y)) \cdot d(x, y), \text { for } x, y \in X \text { with } x \leq y \text {. }
$$

Also suppose,

$$
\begin{gathered}
\text { if }\left\{x_{n}\right\} \subset X \text { is a nondecreasing sequence with } x_{n} \rightarrow z \in X, \\
\text { then } x_{n} \preceq z \text { for all } n \in \mathscr{N} .
\end{gathered}
$$

If there exists an $x_{0} \in X$ with $x_{0} \leq f x_{0}$, then $f$ has a fixed point in $X$.

Corollary 2. Let $(X, \preceq)$ be a partially ordered set and suppose that there exists a metric $d$ in $X$ such that $(X, d)$ is a complete metric space. Suppose $f: X \rightarrow X$ be a nondecreasing mapping and

$$
d(f x, f y) \leq k d(x, y), \text { for } x, y \in X \text { with } x \leq y \text { where } k \in(0,1)
$$

Also suppose,

$$
\begin{gathered}
\text { if }\left\{x_{n}\right\} \subset X \text { is a nondecreasing sequence with } x_{n} \rightarrow z \in X, \\
\text { then } x_{n} \preceq z \text { forall } n \in \mathscr{N} .
\end{gathered}
$$

If there exists an $x_{0} \in X$ with $x_{0} \leq f x_{0}$, then $f$ has a fixed point in $X$.

\section{References}

[1] R. P. Agarwal, M. A. El-Gebeily and D. O'Regan, Generalized contractions in partially ordered metric spaces, Appl. Anal., 87(2008), 109-116.

[2] I. Altun and G. Durmaz, Some fixed point theorems on ordered cone metric spaces, Rend. Circ. Mat. Palermo, 58(2009), 319-325.

[3] A. Amini-Harandi and H. Emami, A fixed point theorem for contraction type maps in partially ordered metric spaces and application to ordinary differential equations, Nonlinear Anal., 72(2010), 2238-2242.

[4] L. Ciric, N. Cakic, M. Rajovic and J. S. Ume, Monotone generalized nonlinear contractions in partially ordered metric spaces, Fixed Point Theory Appl., 2008(2008), Article ID 131294, 11 pages.

[5] T. Gnana Bhaskar and V. Lakshmikantham, Fixed point theorems in partially ordered metric spaces and applications, Nonlinear Anal., 65(2006), 1379-1393.

[6] V. Lakshmikantham and L. Ciric, Coupled fixed point theorems for nonlinear contractions in partially ordered metric spaces, Nonlinear Anal., 70(2009), 4341-4349.

[7] J. Caballero, J. Harjani and K. Sadarangani, Contractive-like mapping principles in ordered metric spaces and application to ordinary differential equations, Fixed Point Theory Appl., 2010(2010), Article ID 916064, 14 pages.

[8] M. Geraghty, On contractive mappings, Proc. Amer. Math. Soc., 40(1973), 604-608.

[9] A. Meir and E. Keeler, A theorem on contraction mappings, J. Math. Anal. Appl., 28(1969), 326-329.

[10] B. Samet and C. Vetro, Coupled fixed point, F-invariant set and fixed point of N-order, Ann. Funct. Anal. $\mathbf{1}(2010), 46-56$. 
Department of Applied Mathematics, National Hsinchu University of Education, Taiwan.

E-mail: ming@mail.nhcue.edu.tw

Department of Applied Mathematics, National Hsinchu University of Education, Taiwan.

E-mail: g9824201@mail.nhcue.edu.tw 